Ophthalmologica

\title{
A Case of Congenital Orbital Malignant Rhabdoid Tumor: Systemic Metastasis following Exenteration
}

\author{
Koung Hoon Kook ${ }^{a}$ Moon Sung Park ${ }^{b}$ Hyunee Yim ${ }^{c}$ Sang Yeul Lee ${ }^{d}$ \\ Jae Woo Jang ${ }^{e}$ Hans E. Grossniklaus ${ }^{f}$

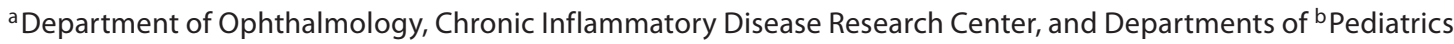 \\ and ${ }^{\mathrm{C} P a t h o l o g y, ~ A j o u ~ U n i v e r s i t y ~ S c h o o l ~ o f ~ M e d i c i n e, ~ S u w o n, ~}{ }^{\mathrm{d}}$ Department of Ophthalmology, Yonsei University \\ School of Medicine, and ' Department of Ophthalmology, Kim's Eye Hospital, Seoul, South Korea; ${ }^{\mathrm{f} D e p a r t m e n t}$ of \\ Ophthalmology, Emory University School of Medicine, Atlanta, Ga., USA
}

\section{Key Words}

Exenteration · Orbital malignant rhabdoid tumor •

Systemic metastasis

\begin{abstract}
A newborn girl presented with massive proptosis of the right eye. Physical and radiologic examination disclosed that the primary orbital mass was confined to the site. A diagnosis of malignant rhabdoid tumor was made by histopathologic examination of an incisional biopsy specimen. Exenteration was performed, and the resection margins were free from tumor cells. However, distant metastasis developed in the liver 1 month after surgery. Despite chemotherapy, the patient died 2 months later due to tumor invasion into the central nervous system, which was confirmed by autopsy. To the best of our knowledge, this is the first case of congenital orbital malignant rhabdoid tumor showing systemic metastasis after exenteration, which suggests the need for aggressive systemic treatment rather than exenteration, even in a case of locally confined tumor.
\end{abstract}

Copyright ๑ 2009 S. Karger AG, Basel
Malignant rhabdoid tumor (MRT) is a rare but highly malignant neoplasm. Since Rootman et al. [1] originally reported primary MRT of the orbit in 1989, there have been several published cases of MRT of the orbit [2-5]. Although MRT characteristically has an early age at onset, MRT occasionally presenting at birth is referred to as congenital. There have been only two published reports of cases with congenital MRT confined to the orbit [6, 7]. Interestingly, both of these patients had a good outcome, showing complete remission after chemotherapy followed by surgical resection and chemotherapy with Gamma Knife radiosurgery, respectively. Therefore, the role of exenteration has not been established for congenital orbital MRT. Here, we describe a case of congenital orbital MRT which was initially treated by exenteration; the patient later developed systemic metastasis and finally succumbed to the tumor despite chemotherapy.

\section{Case Report}

A newborn girl presented with massive proptosis of the right eye, which was identified as secondary to an orbital mass found at 35 weeks of gestation by prenatal ultrasonography (fig. 1a). She was born full term to a healthy mother after a normal pregnancy and weighed 2,590 $\mathrm{g}$ at birth. A large, firm mass completely filled

\section{KARGER}

Fax +4161306 1234 E-Mail karger@karger.ch www.karger.com
Jae Woo Jang

Department of Ophthalmology, Kim's Eye Hospital

156 Youngdeungpo-dong, Youngdeungpo-gu

Seoul 150-034 (South Korea)

Tel. +82 22639 7777, Fax +82 22677 9214, E-Mail greatj@unitel.co.kr 

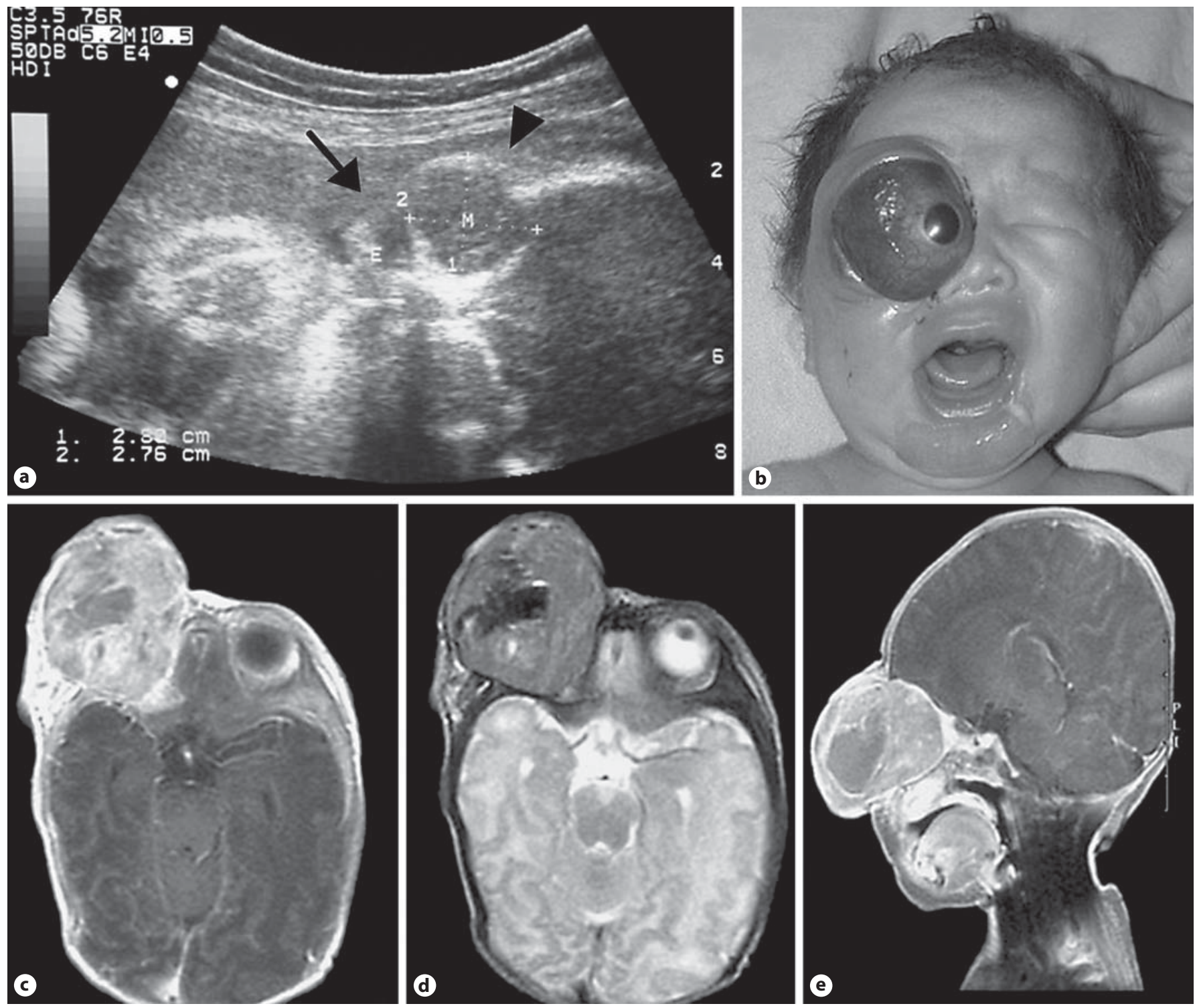

Fig. 1. a Ultrasonography at 35 weeks' gestation, showing orbital mass (arrowhead) and displaced eyeball (arrow). b Clinical photograph taken on day 1 of life, showing a huge mass in the right orbit and proptosis. Axial $\mathrm{T}_{1}(\mathbf{c})$, axial $\mathrm{T}_{2}(\mathbf{d})$, and sagittal $\mathrm{T}_{1}(\mathbf{e})$ magnetic resonance imaging of the orbit after gadolinium injection; well-enhanced and marginated heterogeneous tumor without intracranial extension is seen. the right orbit and was associated with hemorrhage (fig. 1b). The right pupil was fixed and not reactive to light. Magnetic resonance imaging of the head and orbits revealed a large, well-delineated right orbital mass with no evidence of surrounding bony erosion or intracranial extension (fig. 1c-e). The remainder of the physical examination was unremarkable, and the systemic evaluation, including chest X-ray and computed tomography of the chest and abdomen, showed no evidence of a primary nonocular tumor or metastatic disease.

Incisional biopsy of the orbital mass was performed on day 13 of life. Histopathologically, the tumor consisted of an infiltrating small-cell neoplasm that was partially necrotic. Most cells were round with prominent, vesicular nuclei and distinct nucleoli. There were abundant amphophilic or faintly eosinophilic cytoplasmic inclusions (fig. 2a). Immunohistochemical staining was positive for vimentin, cytokeratin, epithelial membrane antigen, and MIC-2 (CD99), but negative for desmin, smooth muscle actin, HMB-45, CD31, CD34, S-100 protein, neuron-specific enolase, and chromogranin A (fig. 2b). All of these findings were consistent with a diagnosis of MRT.

On day 26 of life, exenteration of the right orbit was performed, and the resection margins were free from tumor cells. She re- 

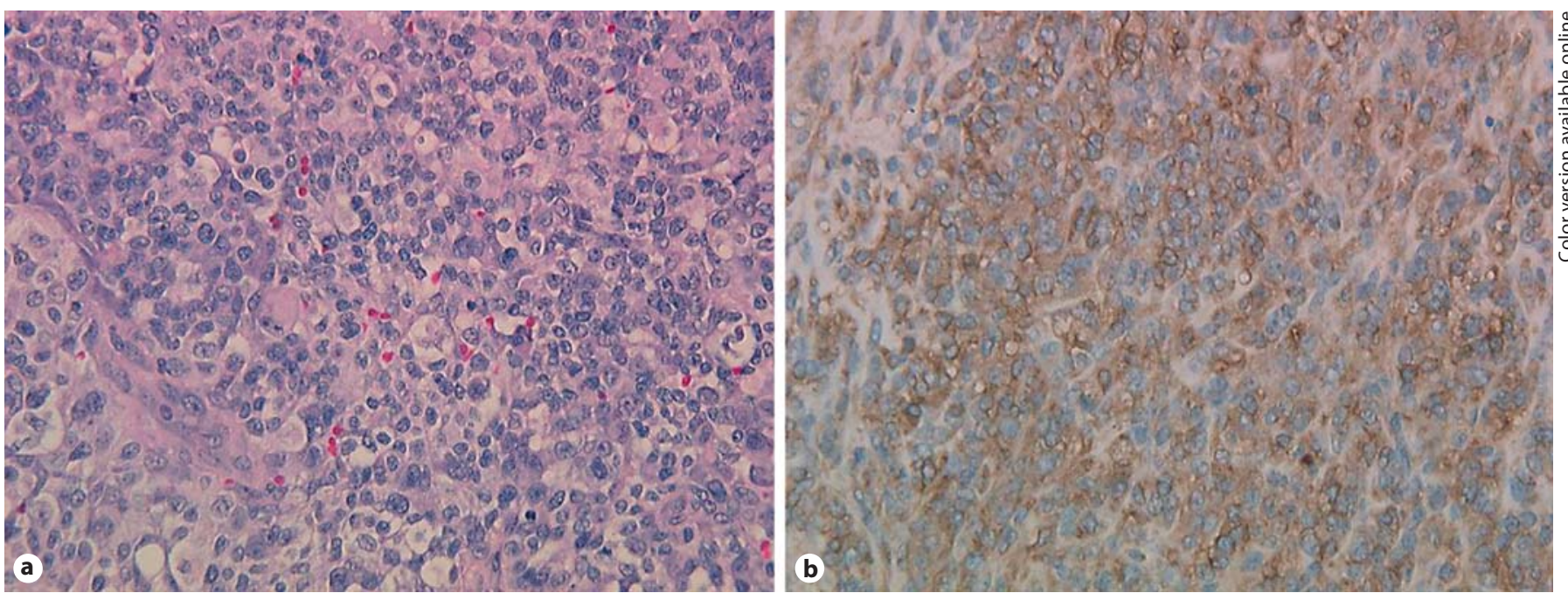

Fig. 2. a Light microscopy image of the orbital tumor, showing sheets of polygonal and spindle cells with vesicular nuclei, prominent nucleoli, and eosinophilic cytoplasmic inclusions. H.E. Original magnification, $\times 400$. b Immunohistochemical analysis showing tumor cells positive for MIC-2 (CD99). Original magnification, $\times 400$.
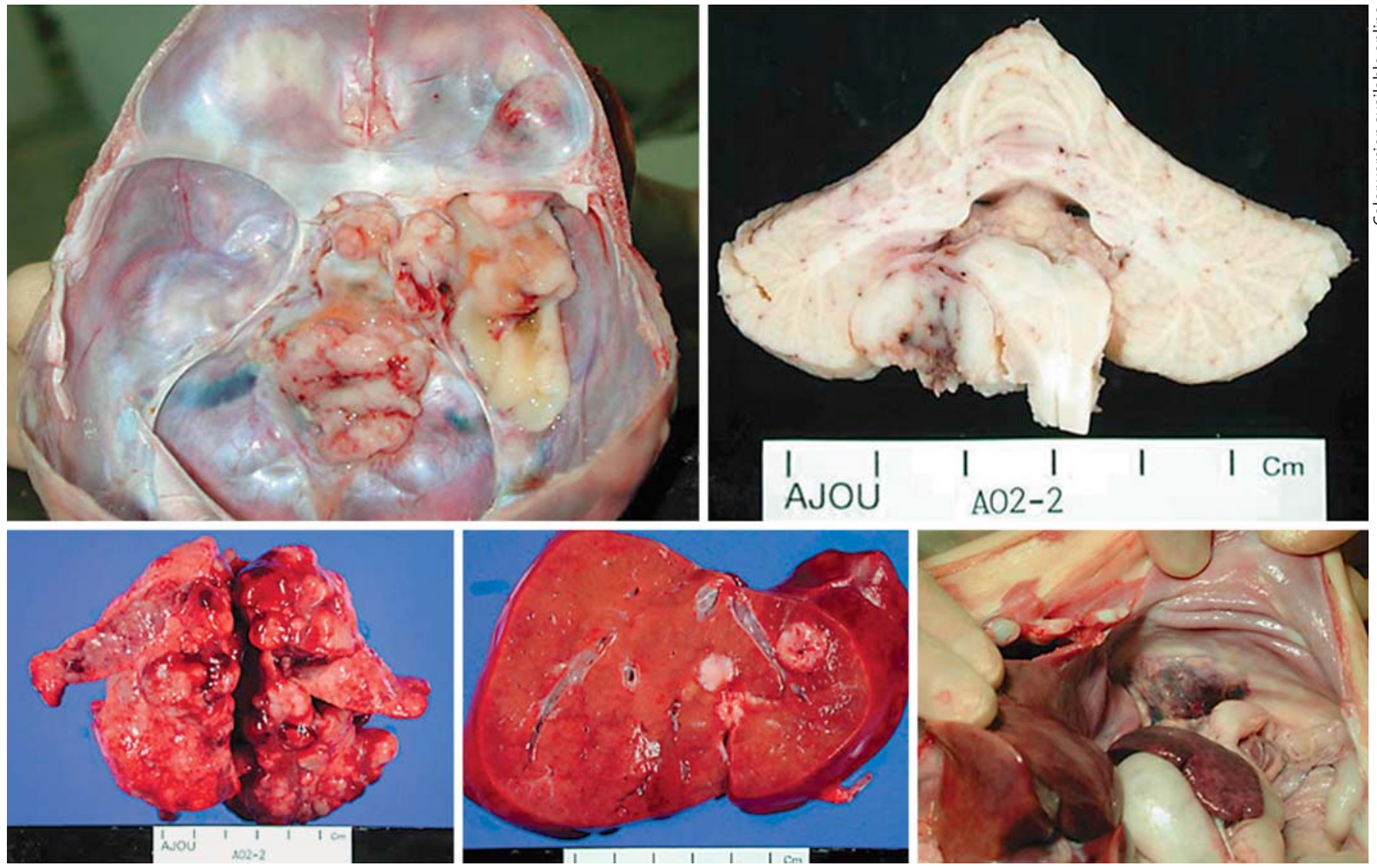

Fig. 3. Autopsy findings of tumor recurrence at exenterated site (top left), pons (top right), posterior aspect of the lung (bottom left), liver (bottom center), and retroperitoneum (bottom right). 
mained in clinical remission for 1 month after surgery. Unfortunately, after this period, multiple hypoechoic nodules, which signified tumor metastasis, were noticed on follow-up abdominal ultrasonography. On that day, chemotherapy was initiated as follows: vincristine $(0.025 \mathrm{mg} / \mathrm{kg})$ on days 1 and 2 , actinomycin D $(0.075 \mathrm{mg} / \mathrm{kg})$ on days $1-5$, and cyclophosphamide $(30 \mathrm{mg} / \mathrm{kg})$ on day 1 . However, at 1 week after the completion of the first cycle of chemotherapy, a computed tomography scan showed multiple, round, hypodense nodules in the lung and liver, which suggested progression of distant tumor metastasis. We recommended a second cycle of chemotherapy with the addition of ifosfamide and etoposide, but her parents refused any further active treatment. Following conservative treatment, the patient died 2 months later. At autopsy, extensive metastatic tumors were observed in the upper portion of the pons, right retro-orbital area, both lungs, chest wall, liver, and retroperitoneum. The masses were firm, irregular shaped, and fixed to adjacent organs (fig. 3).

\section{Discussion}

MRT was initially thought to be a variant of Wilms tumor [8], but it is now known that rhabdoid tumors of the central nervous system (atypical teratoid/rhabdoid tumor), soft tissue (extrarenal MRT), and kidney (rhabdoid tumor of the kidney) have a genetic origin distinct from nephroblastoma [9].

The histopathologic diagnosis of extrarenal rhabdoid tumor is based on the presence of characteristic features similar to those found in kidney MRT. These include large oval to polygonal cells with abundant eosinophilic cytoplasm, large vesicular nuclei with prominent nucleoli, and conspicuous cytoplasmic inclusions. Ultrastructurally, cytoplasmic inclusions are composed of concentric arrays of parallel intermediate filaments, 6-9 $\mathrm{nm}$ in diameter. The filamentous cytoplasmic inclusions are not membrane bound and occasionally incorporate lipid droplets or mitochondria [10]. The differential diagnosis includes round-cell tumors such as rhabdomyosarcoma, neuroblastoma, lymphoma, Ewing sarcoma/primitive neuroectodermal tumor, and malignant melanoma. Immunohistochemistry is helpful in establishing the diagnosis of rhabdoid tumor, which characteristically shows a pattern of positive immunoreactivity for vimentin, cytokeratin, and epithelial membrane antigen. Vimentin is usually positive in rhabdoid tumor, but immunoreactivity for cytokeratin and epithelial membrane antigen is more variable. Negative staining for muscle markers, histiocytic markers, HMB-45, and S100 protein serves to exclude myogenic and histiocytic neoplasms, as well as malignant melanoma [11].

Congenital Malignant Rhabdoid Tumor of the Orbit
The treatment modality for orbital MRT is not yet established, and the role of exenteration is not clear. Interestingly, two previous reports of congenital MRT confined to the orbit showed good prognosis after combined treatment: chemotherapy followed by surgical resection of residual tumor, or Gamma Knife radiosurgery after tumor burden reduction with chemotherapy [6, 7]. Briefly, the former case was treated with three cycles of the ICE (ifosfamide, carboplatin, and etoposide) regimen following monthly intrathecal injections of methotrexate and cytarabine and high-dose chemotherapy in advance of surgical resection of the tumor, and completed by three additional cycles of chemotherapy with the same regimen [6]. The latter case was first treated with a cycle of cisplatin, etoposide, and vincristine. However, because of tumor progression, two cycles of ifosfamide and doxorubicin were included to reduce the tumor burden. After performing Gamma Knife radiosurgery, treatment was completed with three additional cycles of chemotherapy with the same regimen, followed by high-dose chemotherapy with melphalan and cyclophosphamide, and ifosfamide and thiotepa [7].

Exenteration was performed in our patient as a result of the finding that the tumor appeared to be confined to the orbit, with no sign of systemic metastasis. Unfortunately, distant metastasis was found in the liver 1 month after surgery. Although the chemotherapy regimen that we used was not exactly the same as those in the previous cases, despite the chemotherapy, multiple systemic metastases progressed to the lung and liver. The patient died 4 months after initial diagnosis from distant metastasis in the pons and lung, which was confirmed by autopsy.

Although extremely rare, congenital MRT of the orbit should be considered in the setting of rapidly expanding orbital lesions appearing in the neonatal period, and we believe this case suggests the restricted role of exenteration for congenital orbital MRT.

\section{Acknowledgement}

This work was supported by the Korea Science and Engineering Foundation grant (R13-2003-019-01004-0) funded by the South Korean government. 


\section{References}

-1 Rootman J, Damji KF, Dimmick JE: Malignant rhabdoid tumor of the orbit. Ophthalmology 1989;96:1650-1654.

-2 Gunduz K, Shields JA, Eagle RC Jr, et al: Malignant rhabdoid tumor of the orbit. Arch Ophthalmol 1998;116:243-246.

3 Niffenegger JH, Jakobiec FA, Shore JW, Albert DM: Adult extrarenal rhabdoid tumor of the lacrimal gland. Ophthalmology 1992; 99:567-574

4 Johnson LN, Sexton FM, Goldberg SH: Poorly differentiated primary orbital sarcoma (presumed malignant rhabdoid tumor). Radiologic and histopathologic correlation. Arch Ophthalmol 1991;109:1275-1278.
5 Walford N, Deferrai R, Slater RM, et al: Intraorbital rhabdoid tumour following bilateral retinoblastoma. Histopathology 1992 ; 20:170-173.

6 Stidham DB, Burgett RA, Davis MM, Plager DA: Congenital malignant rhabdoid tumor of the orbit. J AAPOS 1999;3:318-320.

7 Watanabe H, Watanabe T, Kaneko M, et al: Treatment of unresectable malignant rhabdoid tumor of the orbit with tandem highdose chemotherapy and gamma-knife radiosurgery. Pediatr Blood Cancer 2006;47: $846-850$.

8 Beckwith JB, Palmer NF: Histopathology and prognosis of Wilms tumors: results from the First National Wilms' Tumor Study. Cancer 1978;41:1937-1948.
9 Gottlieb C, Nijhawan N, Chorneyko K, O'Grady KF, Harvey JT: Congenital orbital and disseminated extrarenal malignant rhabdoid tumor. Ophthal Plast Reconstr Surg 2005;21:76-79.

10 Parham DM, Weeks DA, Beckwith JB: The clinicopathologic spectrum of putative extrarenal rhabdoid tumors. An analysis of 42 cases studied with immunohistochemistry or electron microscopy. Am J Surg Pathol 1994;18:1010-1029.

11 Haas JE, Palmer NF, Weinberg AG, Beckwith JB: Ultrastructure of malignant rhabdoid tumor of the kidney. A distinctive renal tumor of children. Hum Pathol 1981;12: 646-657. 